\title{
Molecular-Dynamics Simulation for Liquid Chromatographic Interactions: Effect of Mobile Phase Composition
}

\author{
Kazuhiro BAN† and Kiyokatsu JINNO \\ Toyohashi University of Technology, School of Materials Science, Toyohashi, Aichi 441-8580, Japan
}

\begin{abstract}
A molecular-dynamics simulation method has been applied to investigate the influence of the mobile-phase composition on the retention of solutes in HPLC. The distribution profiles of the distance between two atoms in ODS ligands were constructed to characterize the conformation of ODS ligand molecules. The distinct difference of ODS conformation is observed by comparing molecular models consisting of solvent molecules at each solvent composition. The distribution profiles of the distance between the mobile-phase solvent molecules and ODS ligand molecules were also constructed to characterize the distribution of the solvent molecules at each composition. In all distribution profiles, the difference in the distribution due to a change in the solvent compositions was very clearly found, and the facts seem to be very reasonable. The distribution profiles of the distance between the solute, $n$-propylbenzene, and the terminal carbon atom in the ODS ligand, and between the solute and the silicon atom in the ODS ligand have been also constructed to see the distribution of the solutes in the separation system. The calculated solute distribution in the ODS-methanol/water system is very consistent with the actual chromatographic retention behaviors.
\end{abstract}

(Received September 29, 2000; Accepted October 18, 2000)

\section{Introduction}

High-performance liquid chromatography (HPLC) is one of the most powerful methods used to separate a wide range of mixtures and to determine a quantity of compounds in many samples. Despite the widespread use of HPLC in both analytical and preparative applications, the separation mechanism has not yet been completely clarified. Therefore, method development and optimization are still often based on empirical "trial and error" approaches. In order to avoid such tedious procedures, several groups have been trying to establish methods to predict retention and to optimize the separation conditions. ${ }^{1-8}$ One of their methodologies is to use various database systems which contain the experimentally obtained retention data. ${ }^{1-4}$ Another approach is the prediction of retention based on Quantitative Structure Retention Relationships (QSRR) and Linear Solvation Energy Relationships (LSER). ${ }^{1,5-8}$ In those approaches, understanding the retention mechanism is the key. Although those works have provided much information about the mechanism, a full understanding of the retention in HPLC is far from being used for retention prediction. In addition, most of those investigations are mainly based on the interaction between the solute and the stationary phase, and some contributions of the mobile phase to the retention mechanism are neglected.

Some computational methods have recently been developed to search for the conformation of proteins and biomolecules. It is well known that the molecular dynamics simulation method (MD method) is one of the most powerful tools to investigate the time-series behavior of a molecular system which contains many molecules. The advances of computer technologies have made it possible to easily handle calculations for a huge

† To whom correspondence should be addressed. molecular system. Recently, some studies have applied this approach to investigate the retention mechanism on reversedphase HPLC, ${ }^{9-16}$ size-exclusion chromatography (SEC), ${ }^{17-19}$ and chiral separation. ${ }^{20-22}$ In this work, an MD simulation was carried out to investigate the influence of the mobile-phase composition to the retention of solutes in HPLC, to discuss the intermolecular distance between the solute and the stationary phase (n-octadecyldimethylsilyl bonded phase, ODS); the conformations of the ODS stationary phase were determined by theoretical calculations.

\section{Experimental}

\section{Molecular modeling}

Two types of molecular models were constructed to investigate the contribution of the mobile phase to the retention. One contains only ODS ligands; the another contains ODS ligands, a mobile-phase solvent mixture of methanol and water, and $n$-propylbenzene as the solute molecule in each molecular system. In this study, the silica gel molecules as the stationary phase support were not contained in the molecular model to reduce the calculation time. Each molecular model is displayed in Fig. 1. In two systems, the number of ODS molecules and the total solvent molecules are fixed as 3 and 54, due to the limitation of the calculation system which we used, respectively. Periodic boundary conditions with the unit-cell dimension $(15 \times 15 \times 30 \AA)$ were applied to generate a mimic of each molecular model. The ODS ligands are arrayed by about a $7.3 \AA$ interval, which was estimated by a calculation based on the ligand density and the surface area of a commercially available ODS column, Discovery C18 (Supelco, PA, USA). The positions of silicon atoms in the ODS ligands are fixed during the MD simulation. The mobile phase-molecules are randomly positioned and the conformation of ODS chains has 

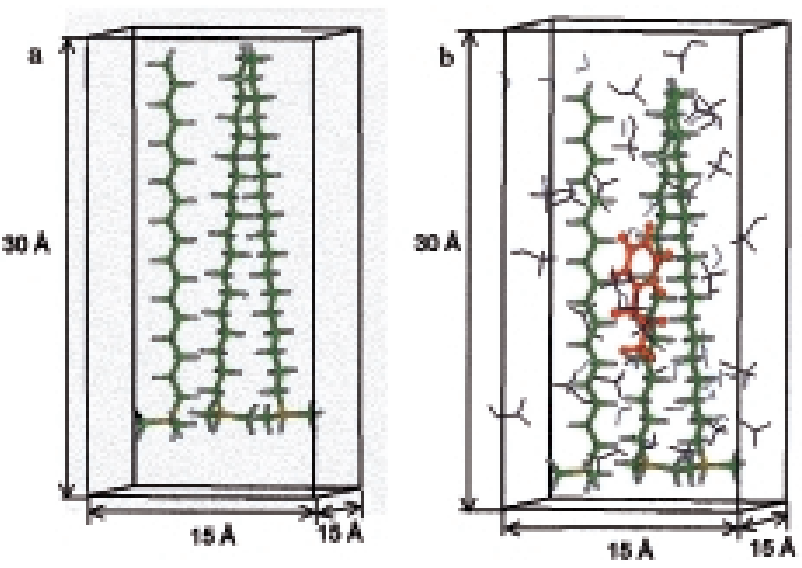

Fig. 1 Initial state of each molecular model used in this study. a, Molecular model containing only ODS ligands: ODS ligand interval, 7.3 A. b, Molecular model containing ODS, mobile phase, and solute n-propylbenzene: ODS ligand interval, $7.3 \AA$ A. Solvent, methanol/water. Total number of solvent molecules, 54 .

Table 1 The number of methanol and water molecules for each solvent composition

\begin{tabular}{ccc}
\hline \multirow{2}{*}{$\begin{array}{c}\mathrm{CH}_{3} \mathrm{OH} / \mathrm{H}_{2} \mathrm{O} \\
(\text { vol \% })\end{array}$} & \multicolumn{2}{c}{ Number of molecules } \\
\cline { 2 - 3 } & $\mathrm{CH}_{3} \mathrm{OH}$ & $\mathrm{H}_{2} \mathrm{O}$ \\
\hline $0 / 100$ & 0 & 54 \\
$20 / 80$ & 5 & 49 \\
$40 / 60$ & 12 & 42 \\
$70 / 30$ & 27 & 27 \\
$80 / 20$ & 34 & 20 \\
$100 / 0$ & 54 & 0 \\
\hline
\end{tabular}

an all-trans conformation orientation in the initial state. In all models, the position of $n$-propylbenzene was determined by the distance between the silicon atom of ODS ligands and the carbon atom of $n$-propylbenzene, which locates at the root position to connect to the propyl group. The mixture of methanol and water was used as the mobile-phase solvent in this work. The composition of the solvent mixture has been varied in $0 / 100,20 / 80,40 / 60,70 / 30,80 / 20$, and 100/0 (methanol/water vol\%). In Table 1, this information is summarized. Computational results were obtained by a software program, Insight II (Molecular Simulation Inc., San Diego, CA, USA). All calculations were performed on an Indigo 2 workstation (SGI, Mountain View, CA, USA).

\section{Computational methods}

In all simulations, the Consistent Valence Forcefield was used to approximate the potential energy of the simulated systems. ${ }^{23}$ The nonbonded interactions for pairs of atoms separated by distances greater than a cutoff value of $5 \AA$ were neglected. To avoid bad contacts arising from the initial modeling process, the energy of each system was first minimized by the steepest descents, followed by the Conjugate Gradients Method, until the derivatives decreased to $0.01 \mathrm{kcal} \mathrm{mol}^{-1} \AA^{-1}$. The systems were then subjected to a constant-volume MD simulation with periodic boundary conditions. The Andersen method was chosen as the temperature-control algorithm, and the temperature of all systems was kept at $298 \mathrm{~K}$ during the simulation. The initial velocities were randomly assigned

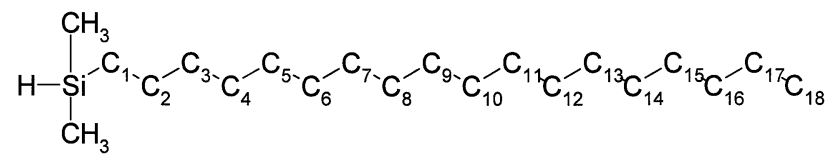

Fig. 2 Basic structure of each $\mathrm{C}_{18}$ bonded phase with the atom numbers used in this simulation work.

according to a Maxwell-Boltzmann distribution for the given temperature. The equations were solved by the Verlet algorithm with an integration time step of 1 fs. ${ }^{11}$ The length of the molecular-dynamics run was 200 ps (200000 time steps). The resulting atomic coordinates were stored on a disk every 50 time steps for a simulation analysis. Following the MD simulation, a number of physical parameters were derived to characterize the molecular properties of each simulated system. In this study, information of the overall position of atoms contained in the molecular model was used to characterize the conformation of the ODS ligand. The information about the distribution of solvent molecules in each molecular model was also used to interpret the results of the MD simulation.

\section{Results and Discussion}

\section{Distribution profiles of the distance between silicon and carbon atoms in ODS ligands}

Following the MD simulation, the distribution profiles of the average distance between silicon atom and carbon atom in three $n$-octadecyldimethylsilyl (ODS) ligands of each simulated molecular system were constructed to obtain information about the conformation of the ODS ligands at each solvent composition. The structure of an ODS ligand is shown in Fig. 2 , where each carbon atom is assigned, as from $\mathrm{C}_{1}$ to $\mathrm{C}_{18}$. The distribution profiles between the silicon atom and the carbon atom in the ligand molecule at each simulated system are shown in Fig. 3, where the peak height indicates the probability of the distribution at each distance during the MD simulation. In Fig. 3a (ODS only), the distributions of each distance were very minimum and the peak of each distribution is very similar to the corresponding distances when the ODS ligand exhibits an alltrans conformation. For a molecular model which contains only water molecules as the mobile-phase solvent, the widest distribution of the distance was observed (Fig. 3b). Especially, increasing the distance between the $\mathrm{Si}$ and $\mathrm{C}$ atoms produced rather extended distribution in the distance. This observation indicates that the twisted conformation exists in this system because of a presence of a repulsive force between the hydrophobic ligand chain and the water molecule. In Fig. 3d (methanol/water $=40 / 60)$, its profile is very similar to the distribution profile of ODS only. Thus, the ordered conformation is predominated under this system. As shown, by increasing the methanol concentration in the solvent composition (Figs. 3e, 3f, and 3g), the overall distance distribution becomes widely extended. These observations might be caused by solvation with the ODS ligand and the methanol molecule; the ligand molecule is twisted due to the bulkiness of the methanol molecule. The results indicate that the existence of the solvent molecule and its composition affect the conformation of the ODS ligand molecule. However, the influence of the difference of the solvent composition on the conformation is not clear. This is due to the fact that the density of the ODS ligands in the simulated system is high (3.0 $\left.\mu \mathrm{mol} / \mathrm{m}^{2}\right)$. The relationship between the density and the 

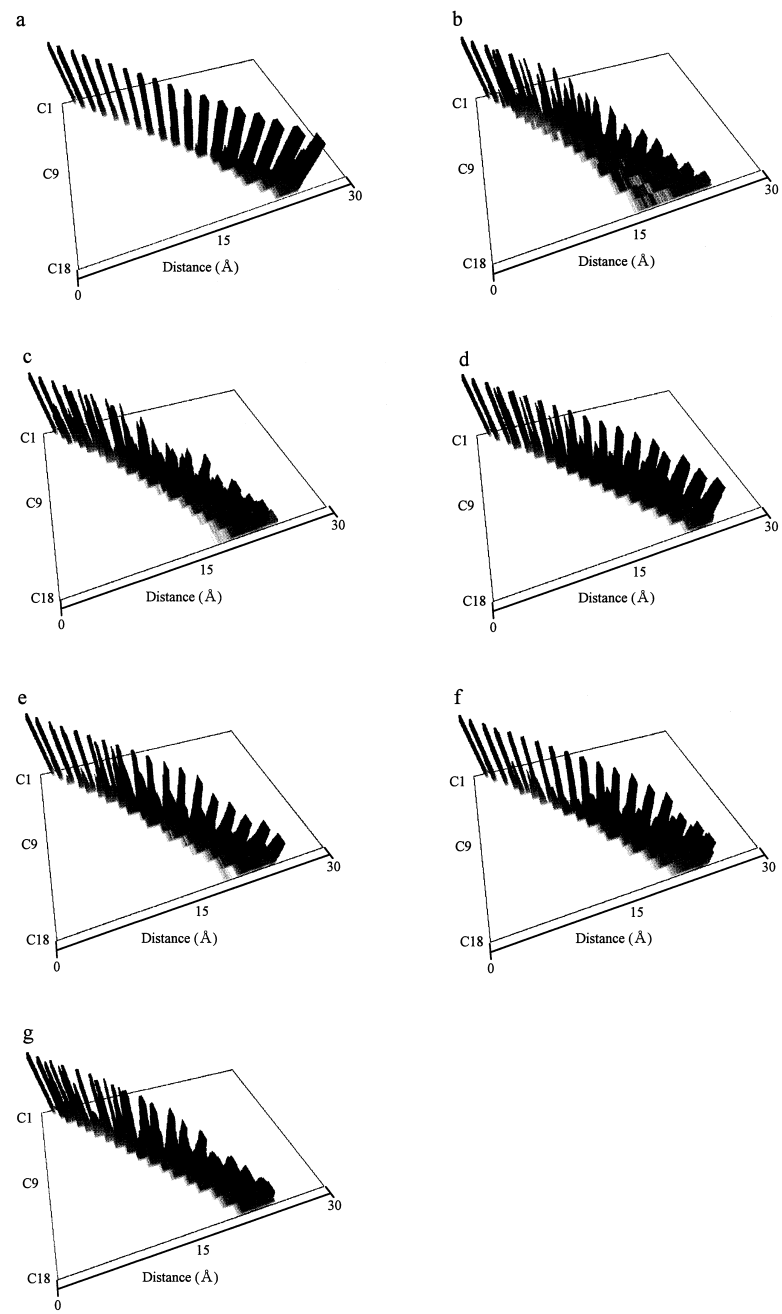

Fig. 3 Distribution profiles of the distance between $\mathrm{Si}$ and $\mathrm{C}_{N}$ atoms $(N=1-18)$ in ODS ligands at each solvent composition. a, no mobile phase solvents; b, 0/100 (methanol/water); c, 20/80; d, 40/60; e, 70/30; f, 80/20; g, 100/0.

conformation of the ligands was reported by Hearn et al.; ${ }^{11}$ this work indicated that the ordered chain conformations were predominated by intermediate and high ligand densities $(2.67$ $\mu \mathrm{mol} / \mathrm{m}^{2}$ and $3.69 \mu \mathrm{mol} / \mathrm{m}^{2}$, respectively).

Distribution profiles of the distance between the ODS ligand and the solvent molecule

The distribution profiles of the distance between the solvent molecules and the $\mathrm{Si}, \mathrm{C}_{9}$, and $\mathrm{C}_{18}$ atoms in the ODS ligand at each solvent composition are shown in Figs. 4, 5 and 6, respectively. For the molecular model at water $100 \%$, the peak of the distribution profile between $\mathrm{Si}$ and water (Fig. 4a) corresponds to the end-to-end distance of the ODS ligand ( 20 $\AA$ ). The distribution profile between $\mathrm{C}_{9}$ and water (Fig. 5a) also corresponds to the $\mathrm{C}_{9}$-to- $\mathrm{C}_{18}$ distance. Moreover, the peak of the distribution profile between the terminal carbon atom $\left(\mathrm{C}_{18}\right)$ and water occurs at $\sim 5 \AA$, although the second broad peak occured at $\sim 10 \AA$, as shown in Fig. 6a. The first peak is likely to be due to a van der Waals interaction between the ligand and water. These results indicate that major water molecules are positioned above the ODS ligand. In contrast, for the molecular model at methanol $100 \%$, a definite peak was observed at $\sim 5 \AA$ in all distribution profiles (Figs. 4f, 5f, and $6 \mathrm{f}$ ) due to the van der Waals interaction between the ligand and methanol molecules.
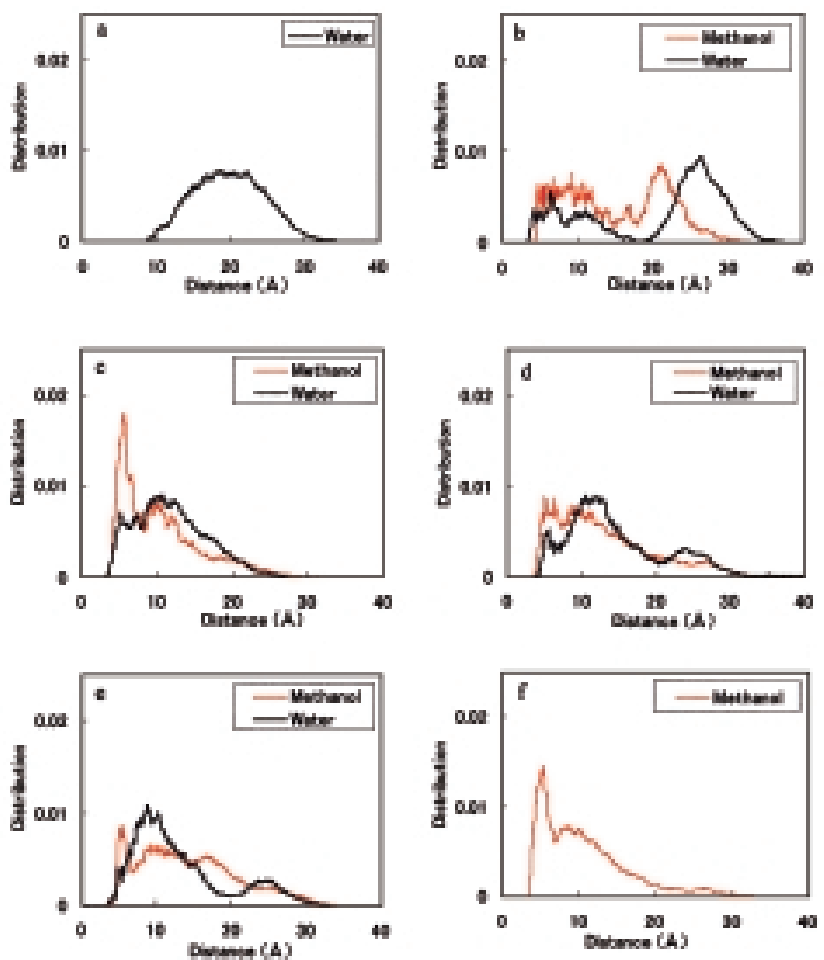

Fig. 4 Distribution profile of the distance between each solvent molecule and $\mathrm{Si}$ atom in ODS with various solvent compositions. a, 0/100 (methanol/water); b, 20/80; c, 40/60; d, 70/30; e, 80/20; f, 100/0.
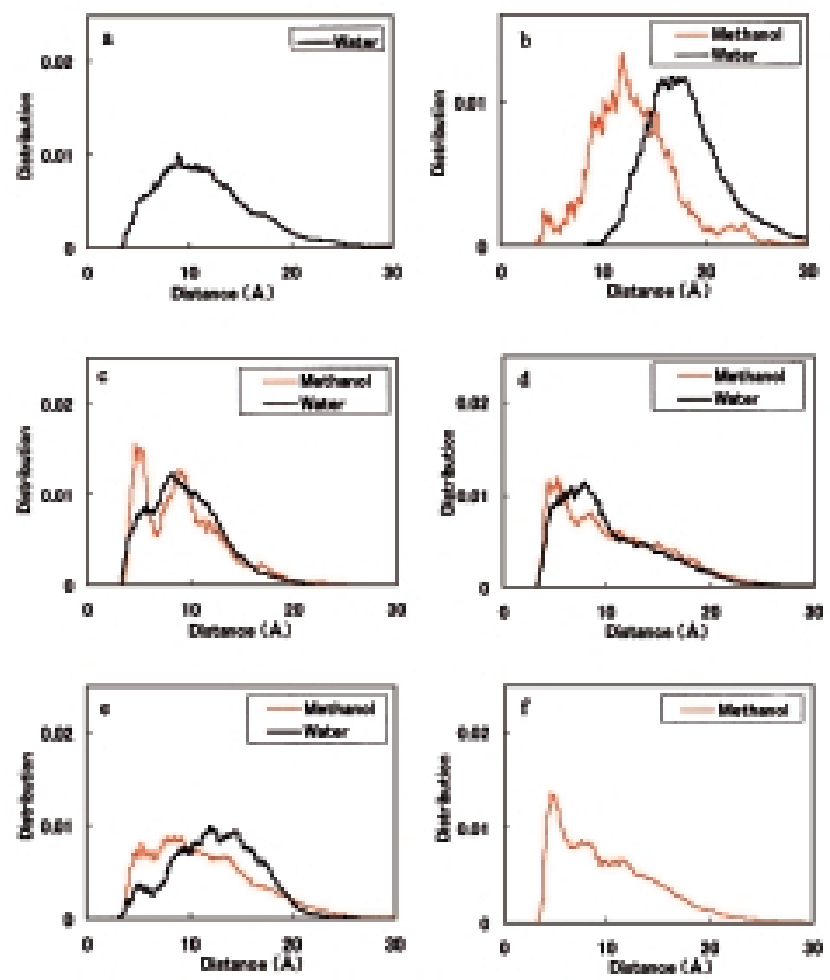

Fig. 5 Distribution profile of the distance between each solvent molecule and $\mathrm{C}_{9}$ atom in ODS with various solvent compositions. a, 0/100 (methanol/water); b, 20/80; c, 40/60; d, 70/30; e, 80/20; f, 100/0.

This observation presents the fact that the ligand is solvated by methanol. 

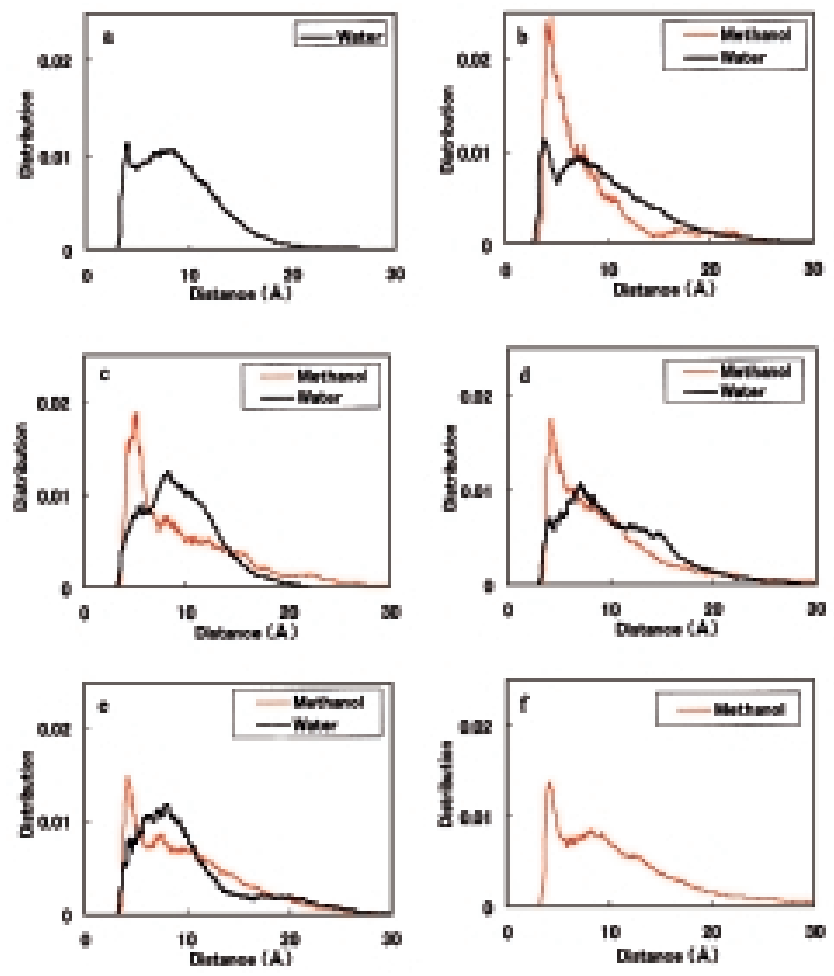

Fig. 6 Distribution profile of the distance between each solvent molecule and $\mathrm{C}_{18}$ atom in ODS with various solvent compositions. a, 0/100 (methanol/water); b, 20/80; c, 40/60; d, 70/30; e, 80/20; f, $100 / 0$.

For the molecular model at methanol/water $=20 / 80$, the methanol distribution has a distinct peak at $7 \AA$, as can be seen in Fig. 6b. The distribution profile of the distance for $\mathrm{Si}$ and water shows a peak at $\sim 27 \AA$, as found in Fig. $4 \mathrm{~b}$. The value of the distance between the ligand and water is larger than that in the case of water $100 \%$; this observation fact indicates that methanol molecules locate very close to the ligand, and that water molecules exist away from the ligand, because the lipophilicity of methanol is stronger than water.

In the case of methanol/water $=40 / 60$, a distinct peak is observed at $\sim 5 \AA$ in all profiles (Figs. $4 \mathrm{c}, 5 \mathrm{c}$, and $6 \mathrm{c}$ ) for the methanol distribution; the values are very similar to those in the case of methanol $100 \%$. This indicates that the ligand molecule is solvated with methanol. In the distribution profile in Fig. 4c, the peak is seen at $\sim 15 \AA$ for water molecules; this indicates that water molecules exist closer than in the case of water $100 \%$ due to the presence of hydrogen bonds with methanol molecules. However, the hydrophobic repulsive force between the ligand and water still exists. For the case of methanol/water $=70 / 30$, the distribution profiles of methanol and water are very similar to each other, as can be seen in Figs. $4 \mathrm{~d}$ and $5 \mathrm{~d}$. This result indicates that both methanol and water molecules are positioned at the similar distance around the ODS ligand molecule.

In Fig. 4e (methanol/water $=80 / 20)$, the peak of the distribution of water-ligand is located at $\sim 10 \AA$; also, the distribution profile of the distance between water and $\mathrm{C}_{18}$ (Fig. 6e) also indicates a similar one at methanol/water $=40 / 60$ found in Fig. 6c. This result indicates that a water molecule forms the hydrogen bond with methanol molecules, which solvate the ODS ligand as well as the case of methanol/water $=40 / 60$.
Table 2 Values of the distance between $n$-propylbenzene, and $\mathrm{C}_{18}$ or $\mathrm{Si}$ atoms in the ODS ligand at the maximum distribution

\begin{tabular}{crc}
\hline & \multicolumn{2}{c}{ Distance/ $\mathrm{A}$} \\
\cline { 2 - 3 } $\mathrm{CH}_{3} \mathrm{OH} / \mathrm{H}_{2} \mathrm{O}$ & $\mathrm{C}_{18}$ & $\mathrm{Si}$ \\
\hline $0 / 100$ & 7.8 & 12.5 \\
$20 / 80$ & 11.0 & 11.3 \\
$40 / 60$ & 10.3 & 11.5 \\
$70 / 30$ & 7.6 & 14.2 \\
$80 / 20$ & 7.1 & 14.2 \\
$100 / 0$ & 6.4 & 19.0 \\
\hline
\end{tabular}

$\mathrm{C}_{18}$ : terminal carbon atom in ODS ligand.

\section{Effect of the solvent composition on the solute position}

The distribution profile of the distance between the solute, $n$ propylbenzene, and the terminal carbon atom $\left(\mathrm{C}_{18}\right)$ in the ODS ligand, and between the solute and the $\mathrm{Si}$ atom in the ODS ligand have also been simulated by using the molecular model. The distance values, which indicate the maximum distribution, are summarized in Table 2. As can be seen, except in the case of $100 \%$ water, increasing the methanol concentration in the solvent mixture induces a decrease in the distance between the solute molecule and the terminal carbon atom $\left(\mathrm{C}_{18}\right)$ in the ODS ligand, and increases of the distance between the solute molecule and the Si atom at the bottom end of the ODS ligand. These behaviors, suggested by the MD simulation, are very consistent with the actual chromatographic retention behaviors in HPLC experiments, where increasing the methanol concentration in the mobile phase decreases the retention.

\section{Conclusion}

An MD simulation has been carried out to investigate the influence of the mobile-phase composition on the retention of $n$ propylbenzene as the test probe in HPLC. Some structural information about the molecular model has been characterized based on the information concerning the conformation of the ODS ligand and the distribution of the mobile-phase solvent molecules in the molecular model. The distribution profiles of the distance between solvent molecules and several atoms in the ODS ligand can be reasonably interpreted by the hydrophobic interaction, hydrogen bonding among the ODS ligand, solvated methanol and water molecules. The solute distribution in the ODS-methanol/water system is very consistent with the actual chromatographic retention behaviors.

\section{References}

1. K. Jinno, "A Computer-Assisted Chromatography System", 1990, Hüthing, Heidelberg.

2. K. Jinno and T. Koizumi, Anal. Chim. Acta, 1981, 133, 457.

3. K. Jinno and H. Noda, Chromatographia, 1984, 18, 326.

4. K. Jinno, Bunseki, 1996, 262, 809.

5. K. Jinno and M. Kuwajima, Chromatographia, 1986, 22, 13.

6. R. Kaliszan, "Quantitative Structure-Chromatographic Retention Relationships", 1987, Wiley, NewYork.

7. M. Ahmed, A. Haj, R. Kaliszan, and A. Nasal, Anal. Chem., 1999, 71, 2976.

8. W. J. Cheong and P. W. Carr, Anal. Chem., 1989, 61, 1524. 
9. R. K. Gilpin, J. Chromatogr., 1993, 656, 217.

5931.

10. A. W. Purcell, M. I. Aguilar, and M. T. W. Hearn, Anal. Chem., 1993, 65, 3038.

11. I. Yarovsky, M. I. Aguilar, and M. T. W. Hearn, Anal. Chem., 1995, 67, 2145.

12. I. Yarovsky, M. T. W. Hearn, and M. I. Aguilar, J. Phys. Chem. B., 1997, 101, 10962.

13. I. Yarovsky, M. I. Aguilar, and M. T. W. Hearn, J. Chromatogr., 1994, 660, 75.

14. S. J. Klatte and T. L. Beck, J. Phys. Chem., 1993, 97, 5727.

15. S. J. Klatte and T. L. Beck, J. Phys. Chem., 1995, 99 , 16024.

16. S. J. Klatte and T. L. Beck, J. Phys. Chem., 1996, 100,

17. R. H. Boyd, Macromolecules, 1996, 29, 1182.

18. E. Bolzacchini, V. Viviana, and R. Lucini, J. Chromatogr. A, 1998, 813, 255.

19. A. M. Striegel, R. D. Plattner, and J. L. Willett, Polym Prepr., 1998, 39, 727.

20. E. Yashima, M. Yamada, Y. Kaida, and Y. Okamoto, $J$. Chromatogr. A, 1995, 694, 347.

21. K. B. Lipkowitz, R. Coner, and M. A. Peterson, J. Am. Chem. Soc., 1997, 119, 11269.

22. T. Beir and H. D. Holtje, J. Chromatogr. B, 1998, 708, 1.

23. "Discover User Guide, Version 2.9.8.", 1996, Molecular Simulation Inc., San Diego, CA. 